EPiC Series in Built Environment
Volume 1, 2020, Pages 491-498
Associated Schools of Construction Proceed-
ings of the 56th Annual International Conference

\title{
Deciphering Why 'Cursed Construction Workers' are More Vulnerable to Occupational Accidents
}

\author{
Mostafa Namian, Ph.D. and Donna Hollar, Ph.D. PE. \\ East Carolina University \\ Greenville, North Carolina \\ Farshid Taherpour and Ebrahim Ghiasvand, Ph.D. \\ Bu-Ali Sina University \\ Hamedan, Iran
}

The construction industry has high rates of accidents, but most can be prevented. To prevent accidents, researchers have demonstrated that workers must first identify workplace hazards. However, workers fail to identify most hazards in construction environments. Previous studies report several factors (i.e., safety attitude) that impact construction workers' ability to recognize hazards. However, the role of superstitious beliefs in safety performance has not been studied. This research investigates how construction workers' superstitious beliefs impact their safety performance. To achieve the objectives, 135 construction workers participated in this study. The workers' safety beliefs and the role of supernatural myths in accident causation perception were studied through use of a validated survey instrument. Results revealed that the greater workers' superstitious beliefs, the more inferior their safety performance. Driven by superstitions, a worker believes that accidents are caused by supernatural powers and workers have little control over their fate resulting in inferior safety performance (i.e., hazard recognition). Poor safety performance, in turn, makes workers more vulnerable to accidents strengthening their superstitious beliefs. Results of this study highlight the role of personal superstitious beliefs in safety performance. Findings are beneficial for construction professionals and practitioners seeking long-lasting and effective interventions to improve safety performance.

Key Words: Construction Safety, Superstitious Beliefs, Hazard Recognition, Safety Attitude

\section{Introduction}

Construction is the most fatal industry among all industries in the United States. In 2017 alone, almost one thousand construction workers lost their lives in the US. In fact, one out of five occupational fatalities occurred in construction while only one out of twenty US workers are employed in construction (BLS, 2017). Most construction safety programs rely on proper hazard recognition and safety risk perception of construction workers. In fact, in order to prevent accidents, an existing hazard 
must be identified, and then proper controls based on the perceived safety risk must be applied. Despite the vital importance of hazard recognition and safety risk perception, more than half of hazards remain unrecognized in the construction workplace, according to previous research (Bahn, 2013; Namian et al., 2016a). Previous studies have reported several factors that impact construction workers' ability to recognize hazards and perceive safety risks (Namian, 2016c). The personal attitude of workers toward safety is among the most influential factors that impact the safety performance of construction workers (Biggs et al., 2007; Namian et al., 2016b). A positive safety attitude denotes that a worker prioritizes safety and establishes safe behavior (Langford et al., 2000). Personal safety attitudes of workers can shape safety culture among workers according to Tam and Fung (2012).

To address safety performance deficiencies and foster safety attitudes, providing safety training has been extensively suggested by previous researchers, practitioners, and regulatory agencies (Wilkins, 2011). However, recent research has revealed that although safety training increases the safety knowledge of construction workers, it does not enhance the personal attitude of workers toward safety (Loosemore \& Malouf, 2019). Therefore, there is a gap in the knowledge and understanding of safety attitudes. This article aims to investigate the role of personal beliefs (i.e., superstitious beliefs) upon which safety attitude is founded, in the safety performance of construction workers.

\section{Research Methods}

Past research has shown that the root causes of occupational accidents in construction can be traced to the unsafe behavior of workers (Haslam et al., 2005). Also, the safety behavior of workers highly depends on personal attitudes, beliefs, and culture (Correll \& Andrewartha, 2000). To sustainably promote safety, personal beliefs have a more influential impact on the safety behavior of workers than having access to advanced safety technology and utilizing advance management systems (Alizadeh, 2005). Therefore, to address the gap in the knowledge, the current study aims to investigate how superstitious beliefs of construction workers impact their safety performance (i.e., hazard recognition). To achieve the research objective, workers on active construction projects in Iran were randomly approached and asked to participate in the study. The superstitious beliefs, hazard recognition, and safety risk perception of the participants were measured and subsequently were statistically analyzed. The details pertaining to the participants, safety performance and superstitious belief measurements are explained in the following sections.

\section{Participants}

Forty-three active construction projects located in five cities in Iran, located in the Middle-Eastern region, were randomly selected as research sites. First, permission to interview workers was sought from each project's management personnel. Over a period of two months in 2019, 135 construction workers were recruited to participate in the study. On average, the participants were 37.2 years old (SD $=6.59)$ and had over 18 years of construction experience $(\mathrm{SD}=7.48)$. The participants were interviewed in person using a questionnaire survey and their superstitious beliefs and safety performance were evaluated based on their interview responses.

\section{Superstitious Beliefs}

There is abundant evidence that safety attitude and personal beliefs play a key role in the safety performance of workers (Haslam et al., 2005). However, there is much debate among researchers if such impact is negative or positive towards safety. For example, studies have shown that religious 
beliefs and trust in a supernatural police force promote compliance of people with regulations resulting in a reduction of risky behavior (Torgler, 2006). In another study conducted among Middle Eastern countries, belief in fate was identified as a major obstacle to implementing safety programs (Loney et al., 2012). In fact, further studies have demonstrated that beliefs can act as both a facilitator of safety behavior and possibly a threat to safety (Kouabenan, 2009). Superstitious beliefs are inevitable traits of human beings which can be found in most cultures (Foster \& Kokko, 2008). For example, eastern cultures count on lucky numbers and astrology to make important decisions (Hernandez et al., 2008). Superstition is defined as "the belief or action of ignorance, trust in magic and chance, or the misconception of the cause of events" (Foster \& Kokko, 2008).

In order to evaluate the participants' superstitious beliefs pertaining to the perception of accidents, a "fatalism scale" developed by Kouabenan (1998) was adopted. The scale consists of nine statements and was slightly modified to address cultural differences represented in the questionnaire as it was developed in a Western country (Peltzer \& Renner, 2003). These statements represent superstitious beliefs with respect to accident perception. For example, the first statement of the instrument is "accidents are due to fate, nothing can be done about it." Then, the participants were asked through the interview to what extent they agree with the statement. Their responses were recorded by the interviewers using a scale of 0 to $10(0=$ strongly disagree and $10=$ strongly agree $)$. The scores corresponding to the participant's responses for all statements were added and reported as a numeric value defined as Superstitious Belief (SB) Index. The range of the SB Index score is 0 (minimum) to 90 (maximum). The SB Index is directly related to the participant's level of superstitious beliefs.

\section{Safety Performance}

In order to evaluate the safety performance of workers, two factors were studied: 1) the number of accidents they were accountable for as a passive indicator and 2) workers' hazard recognition performance as a leading indicator.

To measure the hazard recognition performance of the participants, 24 photographic case images, all captured from local construction projects, were used. The case images were representative of a wide variety of activities and included a wide variety of hazards (see Figure 1). Construction experts evaluated the case images to determine the number of existing hazards exhibited (ranging from 5 to 10 hazards). Four of the 24 case images were randomly selected by the researchers and assigned to each worker to decrease the likelihood of bias related to familiarity of a worker to particular construction activity and the relevant hazards. The hazard recognition performance of a worker for a given case image was determined as the percentage of existing hazards that the worker identified (see Eq. 1). The participant's hazard recognition performance was measured as the average hazard recognition percentage of four case images. A similar approach has been used by different researchers in the past (Albert et al., 2014; Namian et al., 2018a; Namian et al., 2018b). The range of each participant's Hazard Recognition performance measure is from $0 \%$ to $100 \%$.

$$
\frac{\operatorname{hazard}_{n}}{\operatorname{Hazards}_{n}} \times 100
$$

Where: hazard ${ }_{n}$ represents the number of hazards identified by a participant for case image $n$; and Hazards $_{\mathrm{n}}$ represents the total number of unique hazards identified by experts 


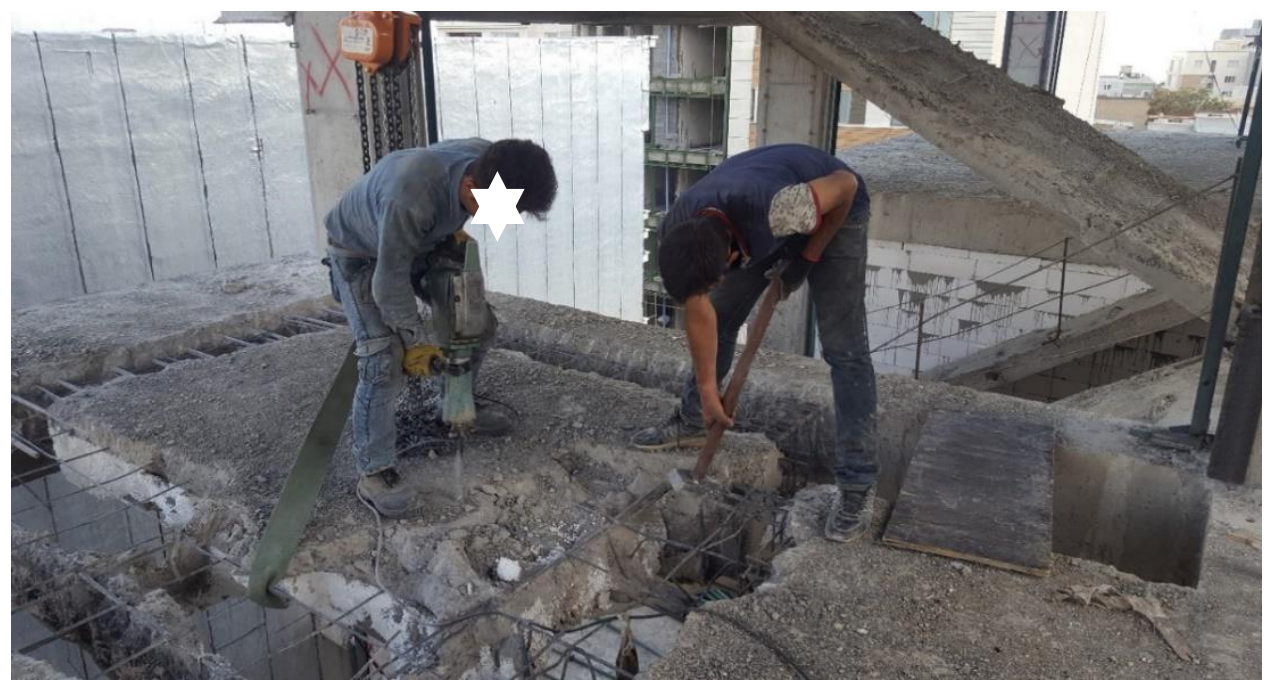

Figure 1. A case image used for workers' hazard recognition and safety risk perception measurement (Image by Farshid Taherpour)

\section{Results and Discussion}

The data collected from the 135 interviewed construction workers were compiled and statistically analyzed. The results show that workers who were accountable for a construction accident in their career had a higher SB Index (SB Index $=60.52$ compared to 50.83); the difference in superstitious beliefs of two groups $(19 \%)$ was significant (p-value <0.0001). In other words, workers who had lower levels of superstitious beliefs reported better safety performance (see Figure 2).

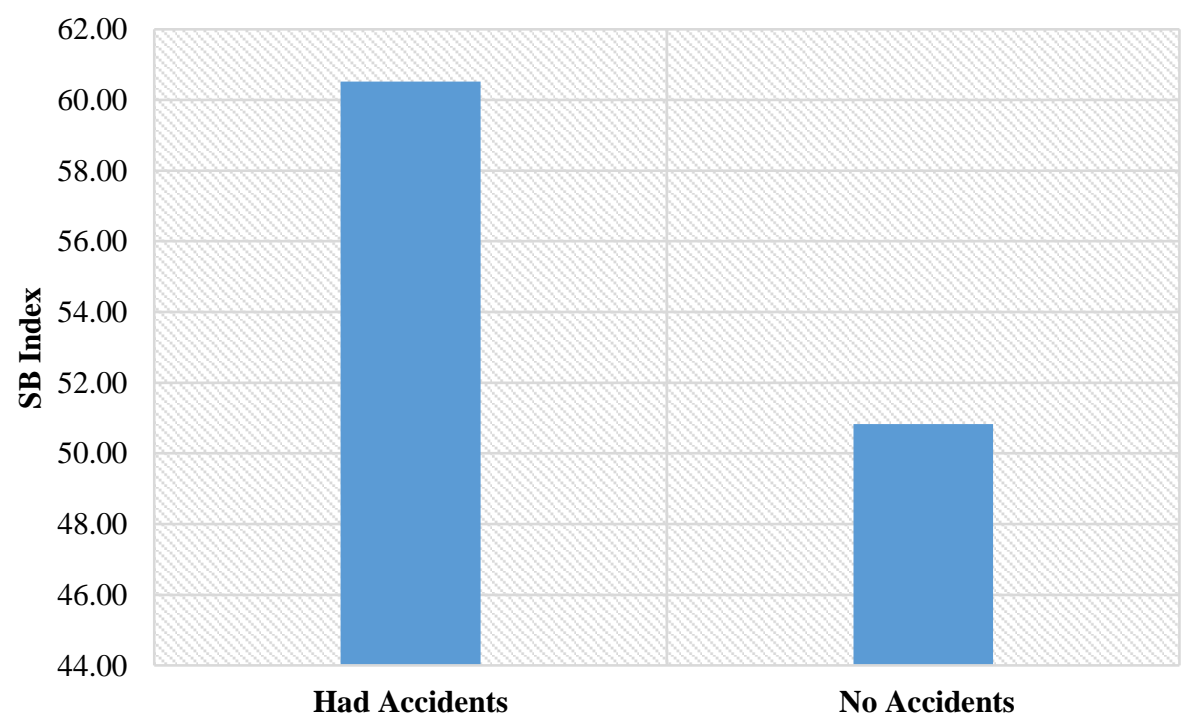

Figure 2. Passive indicator of the safety performance of workers 
Further statistical analysis revealed a significant correlation between hazard recognition and the superstitious beliefs of the participants. The higher a worker's superstitious beliefs (measured by SB index), the less likely the worker is to correctly to identify hazards (see Figure 3). This negative correlation $\left(\mathrm{R}^{2}=0.54\right)$ indicates that having superstitious beliefs (e.g., I had an accident because I was cursed) dictates that the worker has little control over preventing accidents and accidents have supernatural causes; therefore, little effort is directed to identify hazards as the real sources of accidents.

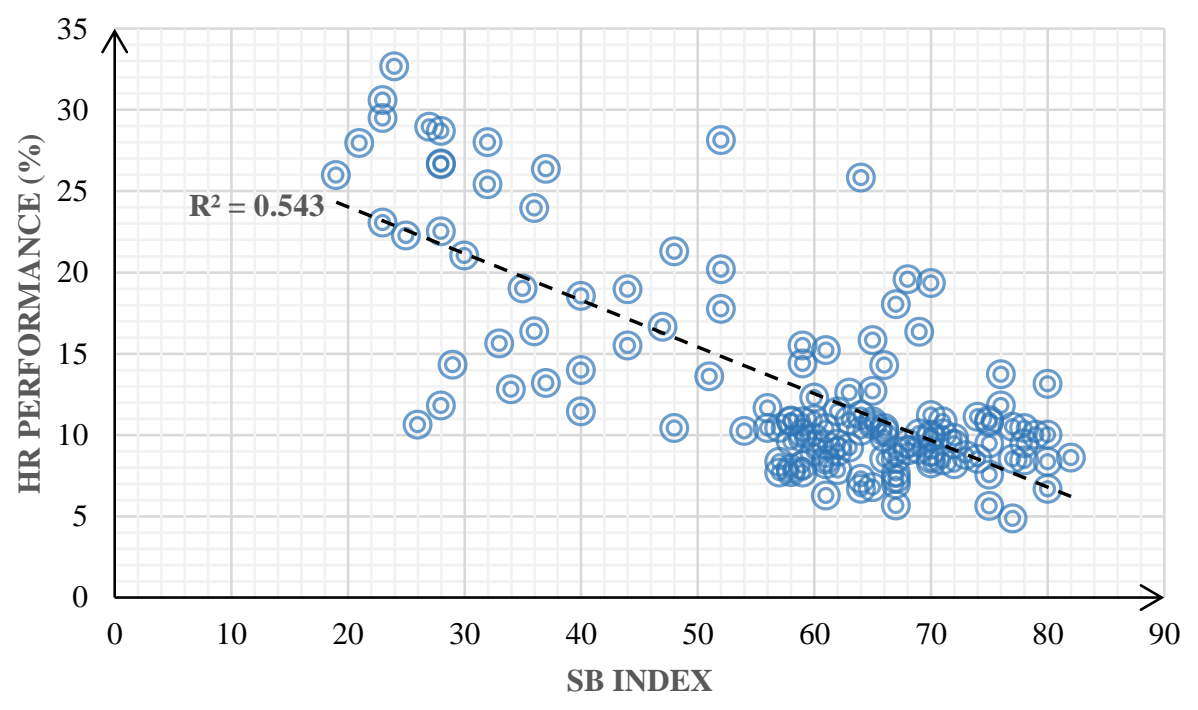

Figure 3. SB Index and hazard recognition performance of workers

The results reveal a harmful behavioral pattern in workers with superstitious beliefs (see Figure 4). Superstitious beliefs discourage workers from identifying hazards in their workplace because the worker believes accidents cannot be prevented due to fate. The likelihood of accidents for a worker who does not attempt to identify, and control hazards increases accordingly. More occurrence of accidents that is not incongruent to superstitious beliefs to such workers. The increase in the occurrence of accidents reinforces irrational beliefs due to observing more strengthening evidence (Biggs et al., 2005).

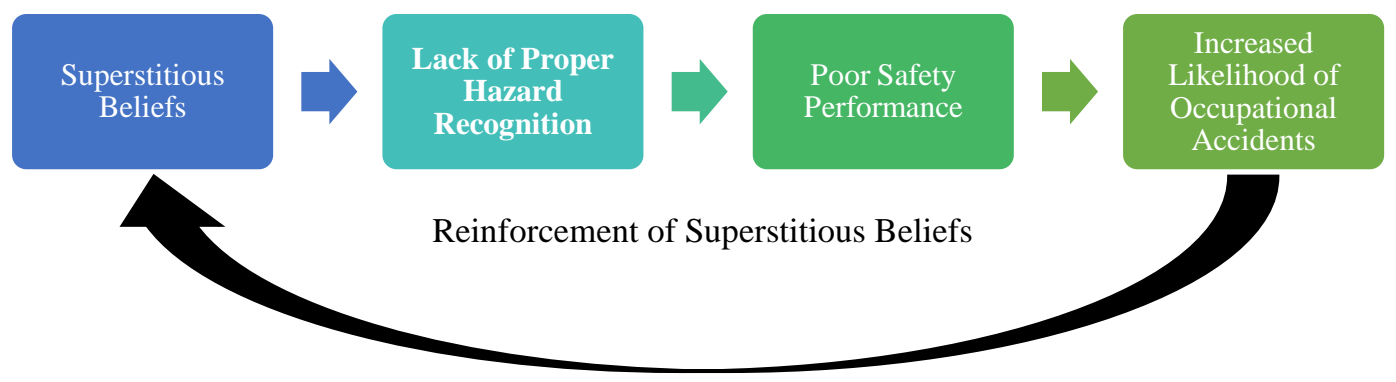

Figure 4. Safety behavior pattern with respect to superstitious beliefs 


\section{Research Implications}

The findings of the current study reveal a safety behavior pattern based on superstitious beliefs. Supported by findings, superstitious beliefs increase the probability of accidents and consequently strengthens the irrational reasoning that accidents due to fate cannot be prevented. Such a loophole in workers' safety behavior is challenging to break. The research findings imply that personal beliefs play a fundamental role in the safety performance of workers. While a worker believes that accidents cannot be prevented, providing personal protective equipment (PPE), enforcing safety rules and regulations, and providing safety training will have little impact. Safety professionals and managers must be aware of their workers' superstitious beliefs which are rooted in culture, religion, and personal experiences. In order to considerably enhance the safety performance of workers, safety interventions must fundamentally address the fallacy in the reasoning of workers based on superstitious beliefs.

\section{Conclusion}

The high rates of construction accidents are attributed to the unsafe behavior of construction workers. Researchers have shown that workers' personal attitudes, beliefs, and cultures impact their safety performance. However, the role of superstitious beliefs in the safety performance of construction workers has not been studied.

The current study aimed to investigate the safety performance of workers who have superstitious beliefs on two safety performance indicators: 1) a passive indicator: accident history and 2) a leading indicator: hazard recognition performance. Researchers have demonstrated that workers who are unable to identify hazards in their workplaces cannot control hazards and prevent accidents.

To achieve the research objectives, 135 construction workers participated in the study. Their demographic information was obtained, their personal beliefs with respect to safety, and their perceived role of supernatural myths in accidents were investigated by adopting a validated survey instrument. Subsequently, the hazard recognition of workers was measured using a validated method.

The results revealed that the higher a worker's superstitious beliefs, the more inferior the worker's safety performance is. In other words, driven by superstitious beliefs, a worker believes people who are injured are a victim of supernatural powers (e.g., they are cursed) and they have little control over their fate. This poor safety attitude, traced to superstitious beliefs, results in inferior safety performance (i.e., hazard recognition). Accordingly, poor safety performance makes the worker more vulnerable to occupational accidents which ultimately strengthens their existing superstitious beliefs.

The results of the current study shed light on the role of personal superstitious beliefs. The findings of this research are beneficial for construction professionals and practitioners who are seeking long-lasting approaches and effective interventions to improve the safety performance of construction workers. Further research is required to explore effective measures to fundamentally change the safety performance of workers by addressing their superstitious beliefs. 


\section{Acknowledgments}

The authors express their appreciation to all managers, workers, and personnel who assisted and/or participated in this research study. This study would have not been accomplished without their help and participation.

\section{References}

Albert, A., Hallowell, M. R., Kleiner, B., Chen, A., and Golparvar-Fard, M. (2014). "Enhancing construction hazard recognition with high-fidelity augmented virtuality." Journal of Construction Engineering and Management, 140(7), 04014024.

Alizadeh, R. (2005). A study on the safety culture and the effect of intervening education based on the BASNEF model and its improvement in the personnel working in production line of Kimia co. Iran medical university, 1-2.

Bahn, S. (2013). "Workplace hazard identification and management: The case of an underground mining operation." Safety science, 57, 129-137.

Biggs, H. C., Sheahan, V. L., \& Dingsdag, D. P. (2005). A study of construction site safety culture and implications for safe and responsive workplaces. The Australian Journal of Rehabilitation Counselling, 11(1), 1-7.

Biggs, H. C., Sheahan, V. L., and Dingsdag, D. P. (2007). "Risk management and injury prevention: competencies, behaviours, and attitudes to safety in the construction industry." The Australian Journal of Rehabilitation Counselling, 13(2), 63-67.

BLS (Bureau of Labor Statistics). (2017). "Census of Fatal Occupational Injuries (Final Data): Industry by Event or Exposure."

Correll, M., \& Andrewartha, G. (2000). Positive safety culture: the key to a safer meat industry. A literature review.

Foster, K. R., \& Kokko, H. (2008). The evolution of superstitious and superstition-like behaviour. Proceedings of the Royal Society B: Biological Sciences, 276(1654), 31-37.

Haslam, R. A., Hide, S. A., Gibb, A. G., Gyi, D. E., Pavitt, T., Atkinson, S., \& Duff, A. R. (2005). Contributing factors in construction accidents. Applied ergonomics, 36(4), 401-415.

Hernandez, M. D., Wang, Y. J., Minor, M. S., \& Liu, Q. (2008). Effects of superstitious beliefs on consumer novelty seeking and independent judgment making: Evidence from China. Journal of Consumer Behaviour: An International Research Review, 7(6), 424-435.

Kouabenan, D. R. (1998). Beliefs and the perception of risks and accidents. Risk Analysis, 18(3), 243252.

Kouabenan, D. R. (2009). Role of beliefs in accident and risk analysis and prevention. Safety Science, 47(6), 767-776. 
Langford, D., Rowlinson, S., and Sawacha, E. (2000). "Safety behaviour and safety management: its influence on the attitudes of workers in the UK construction industry." Engineering, Construction and Architectural Management, 7(2), 133-140.

Loney, T., Cooling, R. F., \& Aw, T. C. (2012). Lost in translation? Challenges and opportunities for raising health and safety awareness among a multinational workforce in the United Arab Emirates. Safety and health at work, 3(4), 298-304.

Loosemore, M., and Malouf, N. (2019). "Safety training and positive safety attitude formation in the Australian construction industry." Safety science, 113, 233-243.

Namian, M., Albert, A., and Feng, J. (2018a). "The Distracted Worker: Effect on Hazard Recognition and Safety Performance." American Society of Civil Engineers, 367-377.

Namian, M., Albert, A., and Feng, J. (2018b). "Effect of distraction on hazard recognition and safety risk perception." Journal of Construction Engineering and Management, 144(4), 04018008.

Namian, M., Albert, A., Zuluaga, C. M., Behm, M. (2016a). "Role of Safety Training: Impact on Hazard Recognition and Safety Risk Perception." J. Constr. Eng. Manage., 142(12), 04016073.

Namian, M., Albert, A., Zuluaga, C. M., and Jaselskis, E. J. (2016b). "Improving hazard-recognition performance and safety training outcomes: Integrating strategies for training transfer." Journal of Construction Engineering and Management, 142(10), 04016048.

Namian, M., Zuluaga, C. M., and Albert, A. (2016c). "Critical Factors That Impact Construction Workers' Hazard Recognition Performance.” Construction Research Congress 2016.

Peltzer, K., \& Renner, W. (2003). Superstition, risk-taking and risk perception of accidents among South African taxi drivers. Accident Analysis \& Prevention, 35(4), 619-623.

Steers, R. M. (1981). Introduction to organizational behavior, Scott Foresman \&Company, Dallas, USA.

Tam, V. W. Y., and Fung, I. W. H. (2012). "Behavior, Attitude, and Perception toward Safety Culture from Mandatory Safety Training Course." Journal of Professional Issues in Engineering Education and Practice, 138(3), 207-213.

Torgler, B. (2006). The importance of faith: Tax morale and religiosity. Journal of economic Behavior \& organization, 61(1), 81-109.

Wilkins, J. R. (2011). Construction workers' perceptions of health and safety training programmes. Construction Management and Economics, 29(10), 1017-1026. 